\section{Detection of myositis-specific antibodies: additional notes}

With great interest, we read the letters that have commented on the recent European League Against Rheumatism/American College of Rheumatology classification criteria for idiopathic inflammatory myopathies ${ }^{1}$ (IIMs) and ensuing discussions. ${ }^{23}$ This highlights how harmonising myositis-specific antibodies (MSA) testing methodologies is currently a hot topic of debate among laboratory specialists, clinicians and manufacturers.

I would particularly like to share our own immunology laboratory experience by expanding on the letter entitled 'Detection of myositis-specific antibodies: additional notes' by M Mahler and M Fritzler ${ }^{4}$ with which we wholly agree.

Newer technologies, such as line immunoassays (LIA) and dot blots (DB), which provide greater sensitivity and rapid serological diagnoses, have standardisation and specificity drawbacks, but the tight connection between autoantibodies and clinical phenotypes underline a great need for improving the test's accuracy. The UK NEQAS external quality assessment reports show clearly how these technologies are steadily increasing in the last years, compared with alternative methods. Though it may seem proficiency testing programmes have shown consistent intermethods agreement results, the data results should be read carefully. Anti-Jo1 positive sera have been analysed very few times as this programme (UK NEQAS for antibodies to nuclear and related antigens) is not focused on anti-Jo1 detection but rather generalised to extractable nuclear antigen (ENA) specificities. Interestingly, there is a very new programme ('Pilot UK NEQAS for Myositis Associated Antibodies scheme') that will consider the anti-Jo1 antibodies in the ENA panel and the total MSA panel. Moreover, as sample selection was mostly limited to single donor serum from a patient with polymyositis, this provided a valid picture of diagnostic sensitivity rather than specificity.

In 2010, Ghirardello et al evaluated the accuracy of commercial LIAs limited to a restricted panel of MSAs showing good global agreement with in-house immunoprecipitation (IP) and immunoblot methods (sensitivity: $47 \%$ vs $51 \%$; specificity $69 \%$ vs $77 \%) .{ }^{5}$ On the other hand, recently, Cavazzana et al assessed the diagnostic accuracy of a new generation LIA by an enlarged panel including a wider spectrum of MSAs that thoroughly addressed the low specificity of LIA/DB methods compared with IP. $^{6}$

Our own laboratory experience reflects how necessary the need to improve specificity performance is, mainly considering the advantages intrinsic to the method, that is, easy to use and inexpensive and its increasing widespread use even in non-specialised laboratories. The sizeable increase in MSA tests has also led to inappropriateness in the IIMs field, similarly to other antinuclear testing in connective tissue diseases (CTDs). As a consequence, a false positive for MSA detection may have a considerable clinical impact: patient anxiety, misdiagnoses, misguided therapies and an unnecessary cascade of insights. Furthermore, a suspected false positive for a cancer-associated MSA (ie, anti-TIF1Y antibodies) may have ethical implications. The open question is: could an inappropriate request in these situations hide the predictive role of the MSAs? Only a strict patient follow-up and a better validation of the test will clarify this point, as there are few data on the predictive role of MSA in the literature.

Given the widespread new technologies and considering that today such a reliable test may allow us early diagnosis of aggressive diseases like those in the IIMs group, it is the manufacturers' and laboratory specialists' duty to strictly validate the LIA/DB tests, especially with regard to more critical antigens, such as anti-SRP and anti-NXP2. Similarly we would like to point out that all critical issues mentioned can be applied to other LIA/DB panels related to other diseases, like scleroderma or other CTDs. We thank the authors for opening the discussion and hope that harmonising the process will improve autoimmunology laboratory work globally.

\section{Maria Infantino, ${ }^{1}$ Mariangela Manfredi, ${ }^{1}$ Valentina Grossi, ${ }^{1}$ Maurizio Benucci ${ }^{2}$}

${ }^{1}$ Laboratory of Immunology and Allergology, S. Giovanni di Dio Hospital, Florence, Italy

${ }^{2}$ Rheumatology Unit, S. Giovanni di Dio Hospital, Florence, Italy

Correspondence to Dr Maria Infantino, Laboratory of Immunology and Allergology, Ospedale San Giovanni di Dio, Florence 55100, Italy; maria2.infantino@uslcentro.toscana.it

Funding This research received no specific grant from any funding agency in the public, commercial or not-for-profit sectors.

Competing interests None declared.

Patient consent Not required.

Provenance and peer review Not commissioned; internally peer reviewed.

(c) Article author(s) (or their employer(s) unless otherwise stated in the text of the article) 2019. All rights reserved. No commercial use is permitted unless otherwise expressly granted.

\section{Check for updates}

To cite Infantino M, Manfredi M, Grossi V, et al. Ann Rheum Dis 2019;78:e29.

Received 27 February 2018

Revised 28 February 2018

Accepted 1 March 2018

Published Online First 13 March 2018

\section{Linked}

- http://dx.doi.org/10.1136/annrheumdis-2018-213341

Ann Rheum Dis 2019;78:e29. doi:10.1136/annrheumdis-2018-213320

\section{REFERENCES}

1 Lundberg IE, Tjärnlund A, Bottai M, et al. European League against Rheumatism/ American College of Rheumatology classification criteria for adult and juvenile idiopathic inflammatory myopathies and their major subgroups. Ann Rheum Dis 2017:69:1955-64.

2 Malaviya AN. 2017 EULAR/ACR classification criteria for adult and juvenile idiopathic inflammatory myopathies and their major subgroups: little emphasis on autoantibodies, why? Ann Rheum Dis 2018;77:e77.

3 Lundberg IE, Tjärnlund A. Response to: '2017 EULAR/ACR classification criteria for adult and juvenile idiopathic inflammatory myopathies and their major subgroups: little emphasis on autoantibodies, why?' by Malaviya. Ann Rheum Dis 2018;77:e78.

4 Mahler M, Fritzler MJ. Detection of myositis-specific antibodies: additional notes. Ann Rheum Dis 2018:annrheumdis-2018-213153.

5 Ghirardello A, Rampudda M, Ekholm L, et al. Diagnostic performance and validation of autoantibody testing in myositis by a commercial line blot assay. Rheumatology 2010:49:2370-4.

6 Cavazzana I, Fredi M, Ceribelli A, et al. Testing for myositis specific autoantibodies: comparison between line blot and immunoprecipitation assays in 57 myositis sera. J Immunol Methods 2016;433:1-5. 